\title{
Apollo asteroid 2005 UD: split nucleus of (3200) Phaethon?
}

\author{
K. Ohtsuka ${ }^{1}$, T. Sekiguchi ${ }^{2}$, D. Kinoshita ${ }^{3}$, J.-I. Watanabe ${ }^{2}$, T. Ito ${ }^{2}$, H. Arakida ${ }^{2}$, and T. Kasuga ${ }^{4}$ \\ 1 Tokyo Meteor Network, Daisawa 1-27-5, Setagaya-ku, Tokyo 155-0032, Japan \\ e-mail: ohtsuka@jb3.so-net.ne.jp \\ 2 National Astronomical Observatory of Japan, Osawa 2-21-1, Mitaka, Tokyo 181-8588, Japan \\ 3 Institute of Astronomy, National Central University, 300 Jhongda Rd, Jhongli, Taoyuan 32001, Taiwan \\ 4 The Graduate University for Advanced Studies, Osawa 2-21-1, Mitaka, Tokyo 181-8588, Japan
}

Received 14 December 2005 / Accepted 7 March 2006

\section{ABSTRACT}

Context. The recently discovered Apollo asteroid 2005 UD is the most likely candidate for being a large member of the Phaethon-Geminid stream Complex (PGC).

Aims. Detecting more complex members like this should clarify the formation and evolution of the PGC.

Methods. Our backward and forward $( \pm 10000$-yr) integration of the Kustaanheimo-Stiefel regularized equation of motion revealed that the orbital evolutions of Apollo asteroids (3200) Phaethon and 2005 UD show a similar profile, time-shifting by $\sim 4600 \mathrm{yr}$.

Results. Within the PGC, this time shift is rather large against the time-lag of $220 \mathrm{yr}$ for Phaethon-Geminids and $\sim 3900 \mathrm{yr}$ between PhaethonSextantids, although much smaller than that of $\sim 19000$ yr between Phaethon-Canis Minorids.

Conclusions. This is a km-order object, hence may be a split nucleus of Phaethon. Besides, the orbital parameters of 2005 UD and the Sextantids are in good agreement along with the time-lag of $100 \mathrm{yr}$. Therefore, the Sextantid meteor shower seems to be associated more closely with 2005 UD than Phaethon.

Key words. minor planets, asteroids - methods: numerical - meteors, meteoroids

\section{Introduction}

Apollo asteroid (3200) Phaethon (=1983 TB) is probably one of the dormant or extinct cometary nuclei from the point of view of the parent of the Geminid meteor stream (Whipple 1983; Ryabova 2001 etc.). In fact, the total mass of the Geminid meteor stream is comparable to those of other major meteor streams of cometary origin (Jewitt 2004). The current orbital parameters of Phaethon are: semimajor axis $(a) \sim 1.27$ AU (astronomical unit); eccentricity $(e) \sim 0.89$, thus perihelion distance $(q) \sim 0.14 \mathrm{AU}$ along with inclination $(i) \sim 22^{\circ}$; however, it has a shorter orbital period of $1.43 \mathrm{yr}$ than any known shortperiod comets.

Before the discovery of Phaethon in 1983, likely multimeteor showers, an association between the Geminids, the Sextantids, and the Canis Minorids, had already been pointed out by Nilsson (1964), Cook (1973), and Kresáková (1974). After the discovery, Babadzhanov \& Obrubov (1987, 1992) simulated the long-term orbital motion of Phaethon, stably changing with a long-period perturbation cycle of $\sim 40000 \mathrm{yr}$. Then a large-amplitude $q-i$ oscillation arises, with a period equal to half the cycle, i.e. $\sim 20000 \mathrm{yr}$, during which the argument of the perihelion $(\omega)$ changes by $\sim 180^{\circ}$. Babadzhanov $\&$ Obrubov also found that Phaethon regularly becomes an Earthcrosser over such a long-term history; consequently they predicted the occurrences of four meteor showers originating in
Phaethon at four different nodal points. After all, Babadzhanov \& Obrubov (1993) successfully reconciled their theoretical meteor shower model with observations. These associated meteor showers are currently observable: Geminids (shower maximum on December 14) and (day time) Sextantids (max. Oct. 2) as active meteor showers; Canis Minorids (max. Dec. 10) and (day time) $\delta$ Leonids (max. Oct. 5?) as rather weak ones. Therefore, the detection of such multi-meteor showers should be strongly evident in the formation of the Phaethon-Geminid stream Complex (hereafter, called PGC), probably formed during Phaethon's active cometary phase long ago. A stream complex formation is considered to be dust particles, released at near perihelion every return from a short-period comet with stable, rather than chaotic, cyclic long-term orbital evolutions, moving away from the comet as time goes by. Eventually, the dust particles should be distributed in and around entire space, drawn by such cyclic orbital changes of cometary motion under planetary perturbations and nongravitational effects. Evidence of the Geminid fireball activities of medieval times (Astapovich \& Terentjeva 1968) should also support such a spatial spread of PGC.

Although a number of attempts to obtain signs of Phaethon's cometary activity have been carried out, no trace of cometary activities has been detected yet (e.g., Hsieh \& Jewitt 2005; Kraemer et al. 2005). The spectral feature of Phaethon is rather bluish, i.e., classified in Tholen's 
Table 1. Orbital parameters of (3200) Phaethon and 2005 UD (equinox J2000).

\begin{tabular}{lccc}
\hline \hline Object & \multicolumn{2}{c}{ (3200) Phaethon } & 2005 UD \\
\hline osculation epoch (TT) & 2005 Aug. 18.0 & -2581 Aug. 18.0 & 2005 Aug. 18.0 \\
mean anomaly $M$ & $115^{\circ} .81866$ & 117.04318 & $353^{\circ} .33143$ \\
perihelion distance $q$ (AU) & 0.1398186 & 0.1534601 & 0.1629124 \\
semimajor axis $a$ (AU) & 1.2713838 & 1.2737898 & 1.2747596 \\
eccentricity $e$ & 0.8900265 & 0.8795248 & 0.8722015 \\
argument of perihelion $\omega$ & $321^{\circ} .99112$ & 207.64364 & $207^{\circ} .46774$ \\
longitude of ascending node $\Omega$ & $265^{\circ} .41395$ & $22^{\circ} .12450$ & $19^{\circ} .84550$ \\
inclination $i$ & $22^{\circ} .17403$ & $28^{\circ} .32959$ & $28^{\circ} .74946$ \\
$\#$ of observations & 1496 & & 287 \\
arc (oppositions) & $1983-2005(19)$ & & Oct. $22-$ Nov. 17 \\
RMS residual & $0^{\prime \prime} .58$ & & $0^{\prime \prime} .25$ \\
absolute mag. $H$ & 14.5 & & 17.5 \\
reference & JPL & this work & Nakano (2005) \\
\hline
\end{tabular}

taxonomy of F-type, as opposed to those of typical cometary nuclei being slightly reddish in general (Tholen 1985; Luu \& Jewitt 1990; Binzel et al. 2004 etc.). The absolute magnitude $(H)$ and the albedo are 14.5 and 0.11 (e.g., Harris 1998), respectively, which is equivalent to a diameter of $\sim 5 \mathrm{~km}$. Other orbital and physical data for Phaethon are summarized on the "Near Earth Objects Dynamic Site" (http://131.114.72.13/cgi-bin/neodys/neoibo) and "Database of Physical and Dynamical Properties of Near-Earth Asteroids" (http://earn.dlr.de/nea/003200.htm).

According to the orbital study by Ohtsuka et al. (1997), Phaethon approaches the Sun up to $27 R_{\odot}$ (solar radius), i.e., $\sim 0.126 \mathrm{AU}$, in the minimum- $q$ phase $\sim 1900 \mathrm{yr}$ ago. Considering its low albedo, the sunny-side surface should be heated over $1000 \mathrm{~K}$, at which temperature the phyllosilicates in $\mathrm{CI}$ and $\mathrm{CM}$ chondrites decompose into olivine, magnetite and anhydrite, etc. (e.g., Nozaki et al. 2006), and the chondritic dust particles may be destroyed (e.g., Mann et al. 2004). Indeed, F-type asteroids, like Phaethon, are regarded as being anhydrous and thermally metamorphosed (Hiroi et al. 1993; Rivkin et al. 2002), and the Geminid meteoroids experienced a thermal history, probably due to the Sun (Halliday 1988; Kasuga et al. 2005). Moreover, a solar thermal stress seems to be a trigger, not only to generate a meteor-stream complex, but also to split and disintegrate a comet such as the 96P/Machholz-Quadrantid stream complex including the near-sun (i.e., sunskirting) Marsden and Kracht comet groups detected by the space-borne coronagraph, SOHO/LASCO (Ohtsuka et al. 2003; Sekanina \& Chodas 2005). Their minimum $q$ reaches up to $\sim 6 R_{\odot}$, then their surface temperature rises at least 1.5 times higher than that of Phaethon. The other sunskirters, Meyer and Kracht II comet groups, along with $q$ of 8-12 $R_{\odot}$ (Meyer 2003; Hoffman \& Marsden 2005; Hönig 2005), would also accompany their complex streams. Therefore, the formation of the PGC may imply the existence of some large members as a split cometary nucleus or fragments among the PGC.

We have long believed our working hypothesis mentioned above, and have been searching for large PGC members. Finally, we found a candidate: a recently discovered Apollo asteroid, 2005 UD.

\section{Orbital evolution of Phaethon}

In the first stage of stream-complex formation, the orbital energy $\left(a^{-1}\right)$ of released meteoroids, fragments, and split nuclei from the parent comet must become slightly different from that of the parent. This results in differences in their evolutional rates. The time-lags (hereafter, called $\Delta t$ ) between orbital evolutions of the parent comet and released matter should be longer with time. Therefore, a large PGC member, if there are any, should be in orbital similarity with Phaethon, shifting by $\Delta t$.

As preliminary work for a PGC survey (mentioned in the next chapter) and for determining the $\Delta t$ of Phaethon with unknown potential PGC members, first of all, we have begun with the orbital evolution of Phaethon. We performed here the backward and forward numerical integration of the KS (Kustaanheimo-Stiefel) regularized equation of motion (cf. Arakida \& Fukushima 2000, 2001) over the term of $10000 \mathrm{BC}$ to $10000 \mathrm{AD}$ (JDT -1931503.5 to JDT 5373 520.5), applying the 12th-order Adams method in double precision with a step size of 0.5 day. We have confirmed that the results of the numerical integration did not significantly change, even when we adopted smaller step size or when we used other integration methods such as the extrapolation method. The initial orbital data of Phaethon at osculation epoch 2005 Aug. 18.0 TT = JDT 2453600.5 were taken from "JPL's HORIZONS System" (http://ssd.jpl.nasa.gov/horizons.html), as listed in Table 1. All the major planets from Mercury through Pluto were included as perturbing bodies, in which the mass of the moon was added to that of the Earth, and the barycenter of their masses was taken as Earth's position. The coordinates of the major planets were taken from the JPL Planetary and Lunar Ephemeris DE408.

\section{Survey}

\subsection{Process}

We surveyed whether there are the large PGC members or not among "the List of Apollo Minor Planets" 

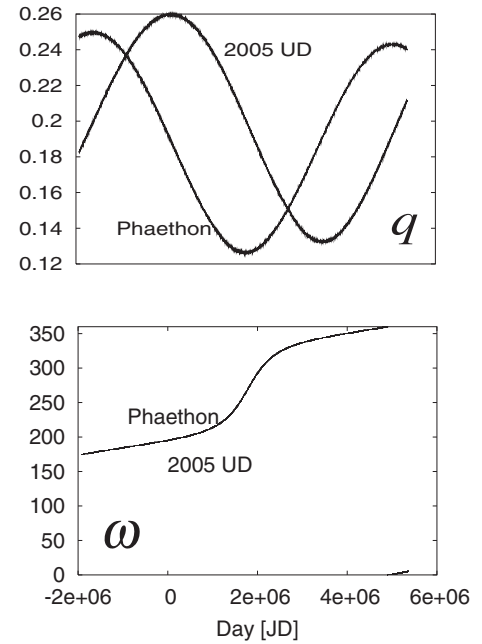
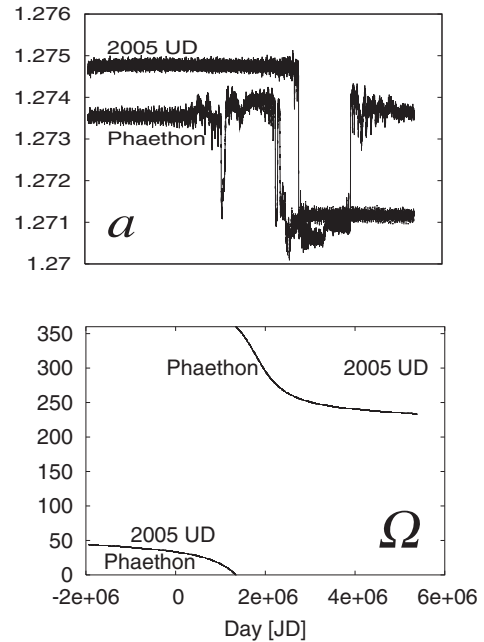
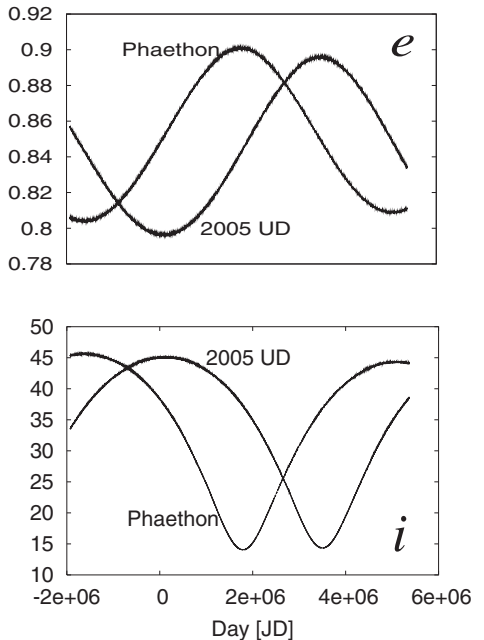

Fig. 1. Orbital evolutions of (3200) Phaethon (thick line) and 2005 UD (thin line), where six graphs show: perihelion distance $q$ in AU (upper left); semimajor axis $a$ in AU (upper center); eccentricity $e$ (upper right); argument of perihelion $\omega$ in degree (lower left); longitude of ascending node $\Omega$ in degree (lower center); and inclination $i$ in degree (lower right). The abscissa of all is time in Julian Terrestrial Date (JDT).

(http: //cfa-www.harvard.edu/iau/mpc.html) and latest MPECs (Minor Planet electric circulars).

As of 2005 November 3, a total of 1758 Apollo asteroids are recorded in the database, among which we directed our attention to 62 Apollos with $q<0.3 \mathrm{AU}$, since $q$ of Phaethon has never been beyond $0.26 \mathrm{AU}$ in our orbital computation. We applied here the following three criteria as the retrieving engine for our survey: the first criterion is traditional $D_{\mathrm{SH}}$ (Southworth \& Hawkins 1963), since in investigating the orbital similarity between two bodies, e.g., comet/asteroid and meteors, we often use $D_{\mathrm{SH}}$. Thus, we traced such a large PGC member on the basis of the Phaethon's orbital evolution from the integration. For each Apollo, we found the minimum $D_{\mathrm{SH}}$ between it and Phaethon, as Phaethon's orbit evolves. When this minimum value of $D_{\mathrm{SH}}$ is below 0.15 , this means that Phaethon and the given Apollo are within the probable association range.

The second and third criteria are $C_{1}$ and $C_{2}$ integrals for the candidate selected by $D_{\mathrm{SH}}$. They were derived by Moiseev (1945) and Lidov (1961), respectively, given as:

$C_{1}=\left(1-e^{2}\right) \cos ^{2} i$,

$C_{2}=e^{2}\left(0.4-\sin ^{2} i \sin ^{2} \omega\right)$.

These integrals describe the secular orbital variations well. Babadzhanov \& Obrubov (1987, 1992) demonstrated that both $C_{1}$ and $C_{2}$ integrals are almost invariant in their $20000-\mathrm{yr}$ backward integration of Phaethon's orbit: $\sim 0.18$ and $\sim 0.27$, respectively. Therefore, $C_{1}$ and $C_{2}$ integrals should also be useful criteria to distinguish a $\mathrm{PGC}$ member.

\subsection{Detection of the candidate: Apollo asteroid 2005 UD}

By these procedures, we finally detected a candidate large PGC member, "Apollo asteroid 2005 UD", recently discovered in the Catalina sky survey on 2005 October 22 (MPEC 2005U22). The orbital elements at epoch 2005 Aug. 18.0
TT $=$ JDT 2453600.5 , listed along with Phaethon in Table 1, were taken from Nakano's (2005) solution, based on 287 positions during an arc of 2005 October 22 to November 17 (27 days) with a rms residual of 0.25 . $H \sim 17.5$ corresponds to a km-order size object. Among these observations, a total of 183 positions along with multi-color measurements were carried out by one of the authors, D. Kinoshita, at Lulin Observatory (1.0-m reflector $f / 8.0+$ CCD) from 2005 October 31 to November 5, when no cometary feature was detected. His results will be published elsewhere as a journal paper.

The current orbital parameters of 2005 UD match those of Phaethon in $2582 \mathrm{BC}$ strikingly well, thus $\Delta t \sim 4600 \mathrm{yr}$. Their $D_{\mathrm{SH}}$ is minimum at only 0.04 , slightly larger compared with the well-established Phaethon-Geminids association of 0.02 (based on the data by Ohtsuka et al. 1997). The $C_{1}$ and $C_{2}$ parameters of 2005 UD fit those of Phaethon: 0.184 and 0.267 , respectively.

Subsequently, using Nakano's data, we also integrated the orbital motion of 2005 UD using the same method as we applied to Phaethon in order to trace both dynamical behaviors of Phaethon and 2005 UD. The solutions of the orbital evolution for both objects are represented in Fig. 1. Phaethon and 2005 UD often closely encounter the terrestrial planets, however, neither of the orbital parameters look chaotic, but rather regular. It is also easy to understand that both orbital evolutions show similar profiles along with quasi-sinusoidal changes, shifting by $\Delta t \sim 4600 \mathrm{yr}$.

\section{Concluding remarks}

We found that Apollo asteroid 2005 UD is the most likely candidate for a large member of the PGC. This is a km-order object, hence may be a split nucleus of Phaethon. We confirmed $\Delta t$ between Phaethon and $2005 \mathrm{UD} \sim 4600 \mathrm{yr}$, which is rather large as against $\Delta t$, being $220 \mathrm{yr}$ for the 
Table 2. 2005 UD, (3200) Phaethon, and the Sextantids (Sekanina 1976) at almost the same evolutional phase.

\begin{tabular}{lccccccc}
\hline \hline Object & $\begin{array}{c}\text { Epoch } \\
\text { (TT) }\end{array}$ & $M$ & $q$ & $e$ & $\omega$ & $\Omega$ & $i$ \\
& & (AU) & & $(2000.0)$ & & \\
\hline 2005 UD & 1867 Aug. 18 & $36^{\circ} .615$ & 0.16570 & 0.87002 & 206.356 & 20.930 & 29.519 \\
Phaethon & -2688 Aug. 18 & $329^{\circ} .118$ & 0.15538 & 0.87800 & 206.759 & 22.996 & $28^{\circ} .956$ \\
Sextantids & 1969 Oct. 9 & $38^{\circ} .0$ & 0.172 & 0.816 & $212^{\circ} .3$ & 15.8 & $31^{\circ} .1$ \\
\hline
\end{tabular}

Phaethon-Geminids association and $\sim 3900$ yr between the Phaethon-Sextantids (Ohtsuka et al. 1997), although much smaller than that of $\sim 19000 \mathrm{yr}$ between the Phaethon-Canis Minorids (Babadzhanov \& Obrubov 1987). Ohtsuka et al. (1997) simply regard $\Delta t$ of the orbital evolutions between Phaethon and presently observable associated meteor streams as an indicator of the meteor stream's age. Ohtsuka et al. also hypothesized that the larger $\Delta t$ means an older age of the meteor stream and more dispersive spatial number density of the meteoroid stream. Really, the hourly rates, i.e., spatial number density of the meteoroid particles, for Geminids, Sextantids, and Canis Minorids are 60, 20, and 2 at most, respectively (Kresáková 1974; Ohtsuka et al. 1997). This suggests that the meteoroid particles are not uniformly distributed over the PGC. Of particular interest should be the relation between 2005 UD and the Sextantids. The orbital parameters of 2005 UD and the Sextantids, picked out by Sekanina (1976) among the Harvard (Havana) radio meteor orbit data, are currently in good agreement. As presented in Table 2, their $\Delta t$ is only $100 \mathrm{yr}$, then $D_{\mathrm{SH}}$ is minimum at 0.08 , while $\Delta t$ between the Phaethon-Harvard Sextantids amounts to $\sim 4700 \mathrm{yr}$, along with somewhat larger $D_{\text {SH }}=0.10$. Therefore, the Sextantid meteor shower seems to be associated more closely with 2005 UD than with Phaethon, judging from $\Delta t$ and $D_{\mathrm{SH}}$.

Another astronomical curiosity is to estimate the splitting time of 2005 UD from Phaethon. Both the dynamical behaviors are closely related with each other. However, $\Delta t$ does not become much larger with time, since there is an imperceptible difference between the two evolutional rates. Such a tendency may cause us difficulty in estimating the splitting time on the basis of our rather short-term orbital solution. In addition, the semimajor axes may have small random changes (similar to those shown in Fig. 1) as a result of perturbations from the terrestrial planets in the long term, and the changes in semimajor axes could then cause changes in precession rates. This problem may be resolved by future work based on further long-term orbital studies.

Acknowledgements. The authors are grateful to Dr. David J. Asher for his constructive comments as the reviewer. Thanks are also due to Prof. Hiroshi Nakai for providing us with useful and detailed information on JPL's Development Ephemeris, DE408. Detailed and constructive review by Yolande McLean has considerably improved the English presentation of this paper. Numerical computations were carried out on a general common use computer system at the Astronomical Data Analysis Center, ADAC, of the National Astronomical Observatory of Japan.

\section{References}

Astapovich, I. S., \& Terentjeva, A. K. 1968, in Physics and Dynamics of Meteors, ed. L. Kresak, \& P. M. Millman (Dordrecht: D. Reidel), 308

Arakida, H., \& Fukushima, T. 2000, AJ, 120, 3333

Arakida, H., \& Fukushima, T. 2001, AJ, 121, 1764

Babadzhanov, P. B., \& Obrubov, Yu. V. 1987, Publ. Astron. Inst. Czechosl., 2, 141

Babadzhanov, P. B., \& Obrubov, Yu. V. 1992, Celest. Mech. Dyn. Astron., 54, 111

Babadzhanov, P. B., \& Obrubov, Yu. V. 1993, Astron. Vest., 27, 110

Binzel, R. P., Rivkin, A. S., Stuart, J. S., et al. 2004, Icarus, 170, 259

Cook, A. F. 1973, in Evolutionary and Physical Properties of Meteoroids, ed. C. L. Hemenway, P. M. Millman, \& A. F. Cook, NASA SP-319, 183

Halliday, I. 1988, Icarus, 76, 279

Harris, A. W. 1998, Icarus, 131, 291

Hiroi, T., Pieters, C. M., Zolensky, M. E., et al. 1993, Science, 261, 1016

Hoffman, T., \& Marsden, B. G. 2005, Sky \& Tel., Aug., 32

Hönig, S. F. 2006, A\&A, 445, 759

Hsieh, H. H., \& Jewitt, D. C. 2005, ApJ, 624, 1093

Jewitt, D. C. 2004, in Comets II, ed. M. C. Festou, H. U. Keller, \& H. A. Weaver (Tucson: Univ. Arizona), 659

Kasuga, T., Watanabe, J., \& Ebizuka, N. 2005, A\&A, 438, L17

Kraemer, K. E., Lisse, C. M., Price, S. D., et al. 2005, AJ, 130, 2363

Kresáková, M. 1974, Bull. Astron. Inst. Czechosl., 25, 20

Lidov, M. L. 1961, Iskusstvennie Sputniki Zemli, 8, 5

Luu, J. X., \& Jewitt, D. C. 1990, AJ, 99, 1985

Mann, I., Kimura, H., Biesecker, D. A., et al. 2004, Space Sci. Rev., 110,269

Meyer, M. 2003, Int. Comet Quart., 25, 115

Moiseev, N. D. 1945, Trudy Gosudarstvennogo Astron. Inst. P.K. Shternberga, 15, 75

Nakano, S. 2005, Apollo asteroid 2005 UD, http://www. oaa.gr.jp/ muramatu/mp/2005ud.htm

Nilsson, C. S. 1964, Aust. J. Phys., 17, 158

Nozaki, W., Nakamura, T., \& Noguchi, T. 2006, M\&PS, in press

Ohtsuka, K., Shimoda, C., Yoshikawa, M., \& Watanabe, J. 1997, EM\&P, 77, 83

Ohtsuka, K., Nakano, S., \& Yoshikawa, M. 2003, PASJ, 55, 321

Rivkin, A. S., Howell, E. S., Vilas, F., et al. 2002, in Asteroids III, ed. W. F. Bottke Jr. et al. (Tucson: Univ. Arizona), 235

Ryabova, G. O. 2001, in Proc. of Meteoroids 2001 Conf., ed. B. Warmbein, ESA SP-495 (Noordwijk: ESTEC), 77

Sekanina, Z. 1976, Icarus, 27, 265

Sekanina, Z., \& Chodas, P. W. 2005, ApJS, 161, 551

Southworth, R. B., \& Hawkins, G. S. 1963, Smithon. Contr. Ap., 7, 261

Tholen, D. J. 1985, IAU Circ., 4034

Whipple, F. L. 1983, IAU Circ., 3881 